\title{
Phase III Study of Enzastaurin Compared With Lomustine in the Treatment of Recurrent Intracranial Glioblastoma
}

\author{
Wolfgang Wick, Vinay K. Puduvalli, Marc C. Chamberlain, Martin J. van den Bent, Antoine F. Carpentier, \\ Lawrence M. Cher, Warren Mason, Michael Weller, Shengyan Hong, Luna Musib, Astra M. Liepa, \\ Donald E. Thornton, and Howard A. Fine
}

See accompanying editorial on page 1097

From the University of Heidelberg, Germany; The University of Texas M.D. Anderson Cancer Center, Houston, TX H. Lee Moffitt Cancer Center, Tampa, FL; Erasmus Medisch Centrum, Rotterdam, the Netherlands; Hopital Pitie Salpetriere, Paris, France; Austin Health, Victoria, Australia; Princess Margaret Hospital and the University of Toronto, Toronto, Ontario, Canada; Eli Lilly and Company, Indianapolis, IN; and Neuro-Oncology Branch, National Cancer Institute, Bethesda, MD.

Submitted March 27, 2009; accepted October 19, 2009; published online ahead of print at www.jco.org on February 1, 2010.

Supported by Eli Lilly and Company.

Presented in part at the 44th Annual Meeting of the American Society of Clinical Oncology, May 30-June 3 , 2008, Chicago, IL.

Authors' disclosures of potential conflicts of interest and author contributions are found at the end of this article.

Clinical Trials repository link available on JCO.org.

Corresponding author: Wolfgang Wick, MD, Department of Neurooncology,

University of Heidelberg, Im Neuenheimer Feld, 400 D-69120 Heidelberg:

e-mail: wolfgang.wick@med.uni-

heidelberg.de.

(C) 2010 by American Society of Clinical Oncology

0732-183X/10/2807-1168/\$20.00

DOI: 10.1200/JCO.2009.23.2595

\section{$\begin{array}{llllllll}A & B & S & T & R & A & C & T\end{array}$}

\section{Purpose}

This phase III open-label study compared the efficacy and safety of enzastaurin versus lomustine in patients with recurrent glioblastoma (WHO grade 4).

\section{Patients and Methods}

Patients were randomly assigned 2:1 to receive 6-week cycles of enzastaurin $500 \mathrm{mg} / \mathrm{d}(1,125-\mathrm{mg}$ loading dose, day 1) or lomustine (100 to $130 \mathrm{mg} / \mathrm{m}^{2}$, day 1). Assuming a $45 \%$ improvement in progression-free survival (PFS), 397 patients were required to provide $80 \%$ power to achieve statistical significance at a one-sided level of .025.

\section{Results}

Enrollment was terminated at 266 patients (enzastaurin, $\mathrm{n}=174$; lomustine, $\mathrm{n}=92$ ) after a planned interim analysis for futility. Patient characteristics were balanced between arms. Median PFS (1.5 $\vee 1.6$ months; hazard ratio $[H R]=1.28 ; 95 \% \mathrm{Cl}, 0.97$ to 1.70$)$, overall survival (6.6 $\vee 7.1$ months; $\mathrm{HR}=1.20 ; 95 \% \mathrm{Cl}, 0.88$ to 1.65$)$, and 6-month PFS rate $(P=.13)$ did not differ significantly between enzastaurin and lomustine, respectively. Stable disease occurred in $38.5 \%$ and $35.9 \%$ of patients and objective response occurred in $2.9 \%$ and $4.3 \%$ of patients, respectively. Time to deterioration of physical and functional well-being and symptoms did not differ between arms ( $H R=1.12 ; P=.54)$. Four patients discontinued enzastaurin because of drug-related serious adverse events (AEs). Eleven patients treated with enzastaurin died on study (four because of AEs; one was drug-related). All four deaths that occurred in patients receiving lomustine were disease-related. Grade 3 to 4 hematologic toxicities were significantly higher with lomustine (46 events) than with enzastaurin (one event; $P \leq .001$ ).

\section{Conclusion}

Enzastaurin was well tolerated and had a better hematologic toxicity profile but did not have superior efficacy compared with lomustine in patients with recurrent glioblastoma.

\section{J Clin Oncol 28:1168-1174. (C) 2010 by American Society of Clinical Oncology}

\section{INTRODUCTION}

Treatment of glioblastoma, a highly lethal brain tumor, ${ }^{1,2}$ includes maximal surgical resection followed by radiotherapy and adjuvant chemotherapy $^{3-5}$ and, recently, temozolomide. ${ }^{6}$ Regardless of initial treatment, all patients experience relapse.

There is no standard systemic therapy available for recurrent glioblastoma, although nitrosoureas are most commonly used at recurrence. ${ }^{7}$ Salvage chemotherapies infrequently result in radiographic and/or clinical improvement and have a limited impact on overall survival (OS) ${ }^{8-10}$ In patients who experience relapse after temozolomide, response rates are low, and only a limited number of nonalkylating agents are available. ${ }^{11}$ Clearly, active, well-tolerated agents that target the underlying molecular abnormalities in gliomas and do not have cross-resistance with alkylating agents are needed.

Gliomas are highly vascular tumors, and the degree of vascularity is associated with malignant progression. ${ }^{12,13}$ Tumor grade and poor prognosis are correlated with vascular endothelial growth factor (VEGF) expression. ${ }^{14}$ VEGFinduced angiogenesis is mediated by protein kinase $C \beta,{ }^{15,16}$ whereas uncontrolled cell growth and chemoresistance are commonly attributed to PTEN loss and phosphatidylinositol-3-kinase (PI3K)/AKT pathway activation. ${ }^{17-19}$ Thus agents 
that target angiogenesis and tumor-dependent signaling pathways are potentially of interest for the treatment of malignant gliomas. ${ }^{20-22}$

Enzastaurin, an oral serine/threonine kinase inhibitor, targets both the protein kinase $\mathrm{C}$ and the PI3K/AKT pathways ${ }^{23}$ to induce apoptosis and suppress proliferation and tumor-induced angiogenesis, as demonstrated in various preclinical models including U87MG glioma cell lines and mouse xenografts. ${ }^{23-25}$ In clinical studies, enzastaurin was well tolerated and has shown encouraging activity in a variety of tumors. ${ }^{26,27}$ In a phase II study of enzastaurin in heavily pretreated patients with recurrent glioblastoma, an interim analysis showed an objective radiographic response rate of approximately $20 \% .^{28}$

On the basis of these encouraging interim data, a randomized phase III study was initiated to compare the efficacy of enzastaurin versus lomustine in recurrent glioblastoma. Lomustine was used as the comparator because nitrosoureas have been most commonly used at recurrence and because an oral agent was preferred. ${ }^{7}$ The primary objective was to compare progression-free survival (PFS). Secondary objectives included comparison of OS, objective response, safety, and patient-reported outcomes. Because this trial was based on results from the interim analysis of a single-site phase II trial, ${ }^{28}$ an interim analysis was planned to determine whether it was scientifically and ethically appropriate to continue enrollment.

\section{PATIENTS AND METHODS}

\section{Eligibility Criteria}

Inclusion criteria included the following: $\geq 18$ years of age; life expectancy $\geq 8$ weeks; Karnofsky performance status (KPS) $\geq 70$; histologically confirmed WHO grade 4 glioblastoma (including gliosarcomas); magnetic resonance imaging (MRI) evidence of tumor progression after radiation and chemotherapy ( $\geq 12$ weeks must have elapsed since completion of radiotherapy or $\geq 4$ weeks for chemotherapy); $\leq 2$ prior chemotherapy regimens; recovery from initial surgery; and adequate organ function. Enzyme-inducing antiepileptic drugs (EIAEDs) were to be discontinued 14 days before study enrollment.

Exclusion criteria included the following: prior enzastaurin therapy within 30 days of enrollment; treatment with any nitrosourea (including lomustine), bevacizumab, investigational drugs, or intratumoral chemotherapy; stereotactic radiosurgery; concurrent systemic anticancer therapy or anticoagulant therapy; second primary malignancy; serious concomitant systemic disorders; ECG or other clinically significant cardiac abnormalities; and pregnancy or breastfeeding.

All patients signed a consent form approved by the participating institution's ethical review board. The study was conducted in accordance with the Declaration of Helsinki and good clinical practices.

\section{Study Design and Treatment Plan}

In this phase III, multicenter, open-label study, patients were randomly assigned $2: 1$ to receive $500 \mathrm{mg}$ of oral enzastaurin daily $(1,125-\mathrm{mg}$ loading dose on day 1) or 100 to $130 \mathrm{mg} / \mathrm{m}^{2}$ of lomustine on day 1 . A 6-week cycle was chosen because the nadir of lomustine at this dose occurs between days 30 and 35. Randomization factors included age, first or second recurrence, and KPS. Treatment continued until disease progression or unacceptable toxicity occurred.

Enzastaurin was omitted for an absolute neutrophil count (ANC) less than $0.5 \times 10^{9} / \mathrm{L}$ for longer than 7 days, an ANC less than $1.0 \times 10^{9} / \mathrm{L}$ with fever (temperature of $101^{\circ} \mathrm{F} / 38.5^{\circ} \mathrm{C}$ ), or a platelet count less than $25 \times 10^{9} / \mathrm{L}$; grade $\geq 3$ transaminase elevations; or grade $\geq 3$ clinically relevant nonhematologic toxicities. If an event resolved to grade $\leq 1$ or patient's baseline, treatment was resumed at $250 \mathrm{mg} / \mathrm{d}$ and re-escalated to $375 \mathrm{mg} / \mathrm{d}$ if the event did not recur for 21 days.
Lomustine was discontinued if treatment could not be administered for 21 days from the time of the next scheduled treatment or if a patient with two prior dose reductions experienced a toxicity that caused a third dose reduction. The dose was reduced by $30 \%$ for an ANC of 1.0 to $1.5 \times 10^{9} / \mathrm{L}$ or platelets 25 to $74.9 \times 10^{9} / \mathrm{L}$ and by $50 \%$ for an ANC less than $1.0 \times 10^{9} / \mathrm{L}$ or platelets less than $25 \times 10^{9} / \mathrm{L}$. Lomustine was restarted after hematologic toxicities resolved to baseline. Lomustine was delayed for grade $\geq 3$ nonhematologic toxicity until resolution to baseline and reduced by $50 \%$ for clinically relevant toxicities. The full dose was resumed if the event did not recur for 42 days after restarting therapy.

\section{Baseline and Treatment Assessments}

Tumor evaluations and neuroradiologic exams using MRI were done after every cycle ( \pm 5 days). Corticosteroid doses had to be stable for 5 days preceding the MRI. All randomly assigned patients (intent-to-treat) were evaluated for efficacy using modified Levin's criteria. ${ }^{29}$ Neuroradiologic data were independently reviewed (central review) to confirm response and progression. Objective response was confirmed by a second MRI more than 4 weeks after the first evidence of response. Subsequent MRI was collected every 6 weeks until progressive disease (PD). Patients had stable disease if no meaningful change was noted in the postcontrast T1 images. PD was defined as increased extent of the enhancing component of evaluable lesions compared with the nadir time point, the appearance of any new lesion, or the worsening of neurologic symptoms.

Patients who discontinued because of PD were evaluated as clinically indicated; those without PD were evaluated every 42 days ( \pm 5 days) until progression or initiation of a new treatment.

Patients who received one or more dose of study drug were evaluated for safety using Common Terminology Criteria for Adverse Events v3.0.

Patients completed the 50-item Functional Assessment of Cancer TherapyBrain (version 4$)^{30,31}$ before random assignment, every 3 weeks, and after discontinuation. Patients with baseline and one or more postbaseline assessments were included in the analysis of time to deterioration $(\mathrm{TtD})$, measured from the date of random assignment to the first date of deterioration in the Trial Outcome Index (TOI; physical and functional well-being plus brain tumor-specific concerns) or death. Deterioration was defined as a decrease from baseline in the TOI that was at least the minimally important difference (11-point decrease on the 148-point TOI scale).

\section{Statistical Methods}

Planned enrollment was 397 patients using a Pocock-Simon minimization algorithm. The baseline factors used in minimization included age ( $<50 v \geq 50$ years), KPS ( $\leq 80 v>80$ ), disease recurrence (yes $v$ no), and institution. The final analysis was planned after 256 PFS events (progression or death), which provided $80 \%$ power to achieve statistical significance at a one-sided level of .025, assuming a 45\% improvement in median PFS of enzastaurin over lomustine.

Survival was determined using Kaplan-Meier estimates and was compared between arms using the log-rank test. The Cox model was used to estimate the hazard ratio (HR), with treatment as the only covariate, and to assess treatment-by-factor interaction for each prespecified baseline factor.

One interim analysis was planned after 107 PFS events to evaluate safety and futility. The futility bound was determined by simulations using a conditional power approach; as guidance, the study would be considered futile if the conditional power under the alternative hypothesis (a 45\% improvement in median PFS) was less than 31\%. Although this was an open-label study, the sponsor was blinded to the aggregate database. Only an independent data monitoring committee had access to the unblinded data.

\section{Pharmacokinetic Assessments}

Plasma samples were collected from patients in the enzastaurin arm on day 1 ( 1 to 5 hours after the first dose of the loading dose) and day 21 of cycle 1 (predose and 3 to 8 hours postdose), or at the time of discontinuation if before day 21 of cycle 1 . If a patient continued on-study for four or more cycles, an additional plasma sample was collected predose on day 1 of cycle 4 . Pharmacokinetic analyses for enzastaurin and its metabolites were conducted at Advion BioServices (Ithaca, NY) using the validated liquid chromatography and tandem mass spectrometry method. 


\section{RESULTS}

\section{Patient Characteristics}

From March 2006 to August 2007, 293 patients entered at 76 sites in 14 countries and 266 were deemed eligible and randomly assigned (Fig 1). Enrollment was stopped at the recommendation of the data monitoring committee after the interim analysis for futility, which was conducted after 115 enrolled patients (43\%) had PD or died.

Patient characteristics were balanced between the two arms (Table 1). The differences observed between arms for sex and age were not statistically significant $(P=.35$ and $P=.39$, respectively). The median time from initial diagnosis to random assignment was numerically longer in the lomustine arm (10.1 v 12.1 months).

\section{Study Drug Administration}

The median duration of therapy for enzastaurin was 42 days (range, 0 to $330+$ days). The mean daily dose for enzastaurin was $513.09 \mathrm{mg}$ (102.6\% of the planned daily dose), excluding the loading dose. Three patients continued to receive enzastaurin for $\geq 1$ year. There were two enzastaurin dose reductions due to cough and thrombocytopenia and six dose omissions (one each for convulsion, edema, nausea, pulmonary embolism, somnolence, and urinary tract infection). Twelve patients discontinued in the enzastaurin arm because of adverse events (AEs), four of which were possibly drug-related serious AEs (erysipelas, aortic thrombosis, cerebral hemorrhage, and seizure).

Patients received a median of one cycle of lomustine (range, one to eight cycles). The mean cycle dose for lomustine was 106.66 $\mathrm{mg} / \mathrm{m}^{2}$ (94.9\% of the planned cycle dose). There were 21 dose reductions (11 because of thrombocytopenia; seven because of neutropenia) and 31 dose delays, mostly due to scheduling conflicts $(\mathrm{n}=16)$ and thrombocytopenia $(\mathrm{n}=10)$. Four patients discontinued lomustine due to drug-related neutropenia, throm-

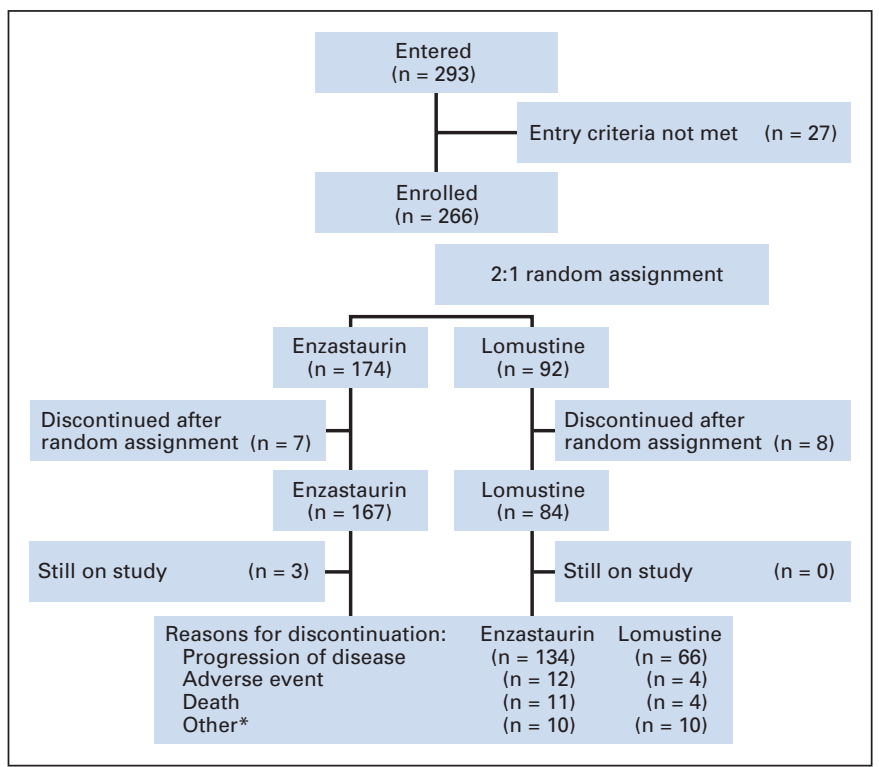

Fig 1. CONSORT flowchart. *Includes protocol violation, entry criteria not met, lost to follow-up, physician or patient decision, or unknown.

\begin{tabular}{|c|c|c|c|c|}
\hline \multirow[b]{2}{*}{ Characteristic } & \multicolumn{2}{|c|}{$\begin{array}{c}\text { Enzastaurin } \\
(\mathrm{n}=174)\end{array}$} & \multicolumn{2}{|c|}{$\begin{array}{l}\text { Lomustine } \\
\text { (n }=92)\end{array}$} \\
\hline & No. & $\%$ & No. & $\%$ \\
\hline \multicolumn{5}{|l|}{ Sex } \\
\hline Male & 116 & 66.7 & 56 & 60.9 \\
\hline Female & 58 & 33.3 & 36 & 39.1 \\
\hline \multicolumn{5}{|l|}{ Age, years } \\
\hline$<50$ & 44 & 25.3 & 28 & 30.4 \\
\hline$\geq 50$ & 130 & 74.7 & 64 & 69.6 \\
\hline \multicolumn{5}{|c|}{ Karnofsky performance status* } \\
\hline Low, 70 or 80 & 83 & 47.7 & 46 & 50.0 \\
\hline High, 90 or 100 & 90 & 51.7 & 45 & 48.9 \\
\hline \multicolumn{5}{|l|}{ Pathologic diagnosis* } \\
\hline Glioblastoma & 171 & 98.8 & 89 & 97.8 \\
\hline Gliosarcoma & 2 & 1.2 & 2 & 2.2 \\
\hline \multicolumn{5}{|c|}{$\begin{array}{l}\text { Time from diagnosis to } \\
\text { random assignment, } \\
\text { months }\end{array}$} \\
\hline Median & \multicolumn{2}{|c|}{10.1} & \multicolumn{2}{|c|}{12.1} \\
\hline Range & \multicolumn{2}{|c|}{$0.9-51.5$} & \multicolumn{2}{|c|}{$4.4-92.0$} \\
\hline \multicolumn{5}{|l|}{ Disease recurrence } \\
\hline First & 129 & 74.1 & 70 & 76.9 \\
\hline Second & 45 & 25.9 & 21 & 23.1 \\
\hline \multicolumn{5}{|l|}{ History of seizures } \\
\hline Yes & 84 & 48.3 & 45 & 48.9 \\
\hline No & 90 & 51.7 & 47 & 51.1 \\
\hline \multicolumn{5}{|l|}{ Prior therapies } \\
\hline Surgery & 174 & 100 & 91 & 98.9 \\
\hline Radiotherapy & 174 & 100 & 91 & 98.9 \\
\hline Systemic therapy & 173 & 99.4 & 90 & 97.8 \\
\hline Corticosteroid & 92 & 52.9 & 48 & 52.2 \\
\hline EIAED therapy & 54 & 31.0 & 25 & 27.2 \\
\hline
\end{tabular}

Abbreviation: EIAED, enzyme-inducing antiepileptic drugs.

*The status of two patients was unknown.

tDisease recurrence was reported in 91 patients in lomustine arm.

bocytopenia, increased transaminase, and non-drug-related deep vein thrombosis (one patient each).

\section{Efficacy}

As shown in Figure 2A, no significant difference was seen between median PFS of enzastaurin (1.5 months) and lomustine (1.6 months, $P=.08$; $\mathrm{HR}=1.28 ; 95 \% \mathrm{CI}, 0.97$ to 1.70 ), with $10.3 \%$ and $23.9 \%$ patients censored, respectively. The 6-month PFS rate was $11.1 \%$ for enzastaurin and $19.0 \%$ for lomustine $(P=.13)$. The median OS of enzastaurin (6.6 months, 30.5\% censoring) and lomustine (7.1 months, $35.9 \%$ censoring) was not significantly different $(P=.25$; $\mathrm{HR}=1.20 ; 95 \% \mathrm{CI}, 0.88$ to 1.65 ; Fig $2 \mathrm{~B}$ ). The median follow-up time for surviving patients was 8.7 months.

Patients with a high KPS (90 to 100) had better PFS (2.8 months $v$ 1.5 months; $P=.007)$ and longer OS (10.3 months $v 7.4$ months; $P=.020$ ) with lomustine. None of the other baseline random assignment factors had a significant effect on overall efficacy. Treatment interaction with time from initial diagnosis to random assignment was not significant for either PFS $(P=.40)$ or OS $(P=.81)$. There was no significant difference in objective response $(P=.501)$ (Table 2$)$.

The consistency rate in the determination of PD between investigator and independent review of scans from 220 patients was $86 \%$ 


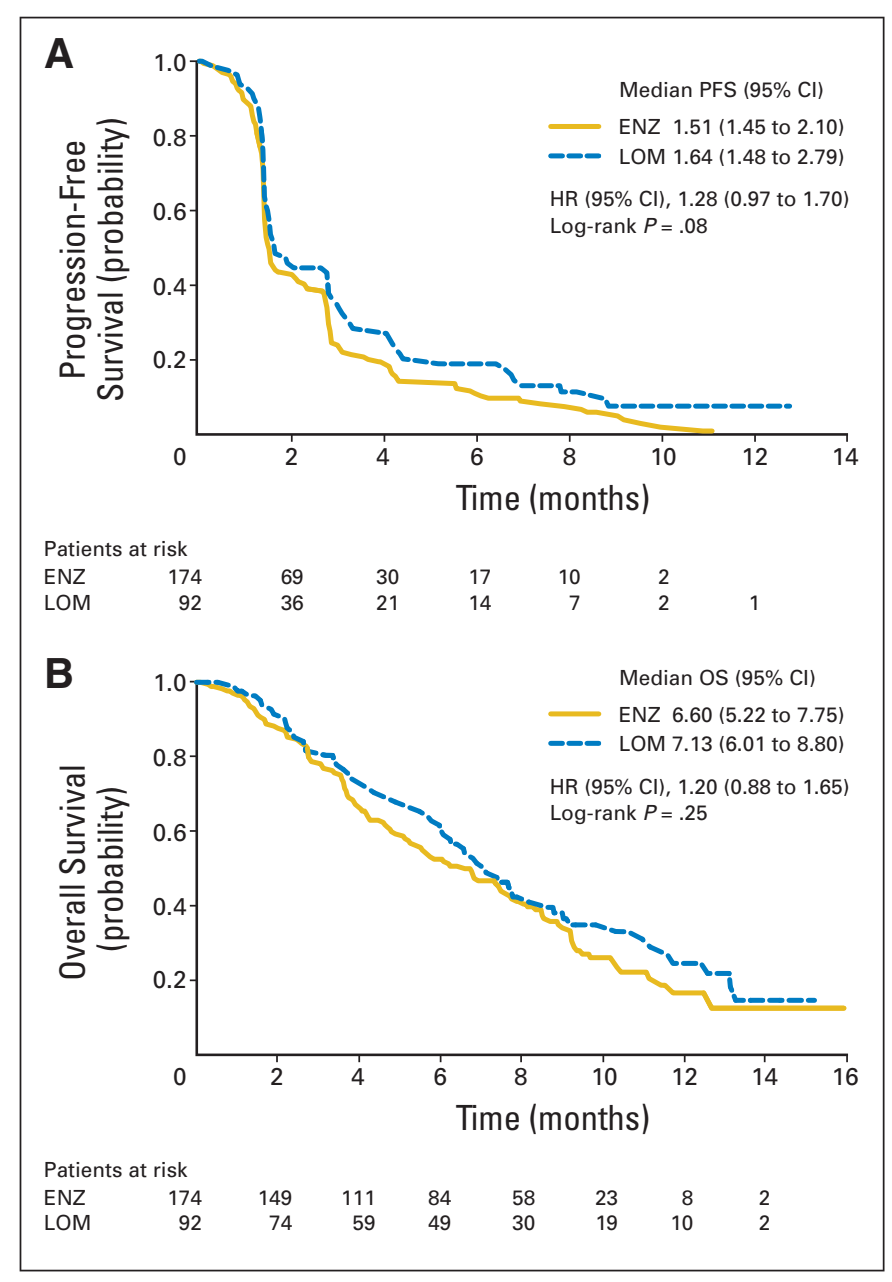

Fig 2. (A) Progression-free survival (PFS) by treatment arm for the intent-to-treat population ( $n=266$ ). PFS was defined as the time from the date of random assignment to the first date of progressive disease, which was identified by magnetic resonance imaging, evidence of neurologic progression, or death. (B) Median overall survival (OS) and $95 \% \mathrm{Cl}$ by treatment arm for the intent-to-treat population ( $n=266$ ). OS was defined as the time from the date of random assignment to death. Time-to-event estimates were calculated using the KaplanMeier method and were compared between regimens using the log-rank test. ENZ, enzastaurin; LOM, lomustine; HR, hazard ratio.

and did not impact the PFS analysis ( $\mathrm{HR}=1.30 ; 95 \% \mathrm{CI}, 0.98$ to 1.73 ). Scans from 50 patients were also assessed by Macdonald's criteria. Consistency rates were $77.5 \%$ and $70 \%$ in the enzastaurin and lomustine arms, respectively.

\section{Patient-Reported Outcomes}

On-study compliance for completion of the Functional Assessment of Cancer Therapy-Brain was $76.7 \%$ in the enzastaurin arm and $78.3 \%$ in the lomustine arm. ${ }^{32} \mathrm{TtD}$ analysis was based on 159 patients treated with enzastaurin (91.4\%) and 82 patients treated with lomustine $(89.1 \%)$. Baseline scores for the TOI were not statistically different between arms $(P=.64)$, with physical well-being scores of 21.4 and 21.3 (of 28), functional well-being scores of 15.5 and 15.4 (of 28), and brain tumor-specific concern scores of 59.8 and 59.1 (of 92), respectively, for enzastaurin and lomustine. Median TtD in patients who had a baseline TOI assessment was 2.27 months for enzastaurin and 2.33 months for lomustine, with 6-month deterioration rates of $18 \%$ and

\begin{tabular}{|c|c|c|c|c|c|}
\hline \multirow[b]{2}{*}{ Overall Radiologic Response* } & \multicolumn{2}{|c|}{$\begin{array}{c}\text { Enzastaurin } \\
(\mathrm{n}=174)\end{array}$} & \multicolumn{2}{|c|}{$\begin{array}{l}\text { Lomustine } \\
\qquad(\mathrm{n}=92)\end{array}$} & \multirow{2}{*}{$\begin{array}{l}\text { Fisher's } \\
\text { Exact } P+\end{array}$} \\
\hline & No. & $\%$ & No. & $\%$ & \\
\hline Objective response & 5 & 2.9 & 4 & 4.3 & .501 \\
\hline Stable disease & 67 & 38.5 & 33 & 35.9 & .692 \\
\hline Progressive disease & 72 & 41.4 & 38 & 41.3 & 1.000 \\
\hline Unknown & 30 & 17.2 & 17 & 18.5 & .866 \\
\hline \multicolumn{6}{|l|}{ Duration of response, $\neq$ months } \\
\hline Range & \multicolumn{2}{|c|}{$4.14-9.63$} & \multicolumn{2}{|c|}{$2.79-9.62$} & - \\
\hline \multicolumn{6}{|c|}{$\begin{array}{l}\text { *Objective response was defined as decreased or no enhancement on the } \\
\text { post-contrast T1 images and stability or improvement on fluid-attenuated } \\
\text { inversion recovery/T2 images. } \\
\text { †Comparison between regimens was based on unadjusted normal distribu- } \\
\text { tion approximation using the Fisher's exact test. } \\
\text { ‡Duration of response was measured from the date when the criteria were } \\
\text { first met for response until the first date of progressive disease or death from } \\
\text { any cause, whichever occurred first. }\end{array}$} \\
\hline
\end{tabular}

$29 \%$, respectively $(P=.54 ; \mathrm{HR}=1.12 ; 95 \% \mathrm{CI}, 0.77$ to $1.63 ; 47 \%$ censoring). ${ }^{32}$ The Pearson correlation coefficient ${ }^{33}$ of TtD with PFS was 0.42 and Kendall's $\tau$ was $P=.001$. The changes from baseline for physical and functional well-being and for brain tumor-specific concerns were not significantly different between arms $(P>.05)$, with physical and functional well-being and brain tumor-specific concern scores worsening by $\leq 2.5$ each over four cycles.

\section{Safety}

Fifteen patients (seven assigned to enzastaurin and eight assigned to lomustine) discontinued before receiving treatment and were not included in the safety analysis (Table 3). The incidence of AEs was similar in both arms; however, $62 \%$ of events in lomustine were drug-related, compared with $44 \%$ in enzastaurin $(P=.008)$. The most common AEs observed were headache in 34 patients (20.4\%), convulsion in 25 patients $(15.0 \%)$, and fatigue in 29 patients $(17.4 \%)$ in the enzastaurin arm and thrombocytopenia in 37 patients $(44.0 \%)$, fatigue in 20 patients $(23.8 \%)$, and nausea in 19 patients $(22.6 \%)$ in the lomustine arm.

Hematologic toxicities were significantly higher with lomustine than with enzastaurin $(P \leq .001)$, including drug-related grade 3 to 4 thrombocytopenia, neutropenia, and leukopenia $(P \leq .007)$. Thrombocytopenia $(n=1)$ was the only grade 3 to 4 hematologic toxicity for enzastaurin.

Significantly more patients treated with lomustine received transfusions $(11.9 \% v 0.6 \% ; P<.001)$. There was no difference in the number of patients hospitalized $(P \geq .247)$.

Eleven patients $(6.6 \%)$ receiving enzastaurin died on study: four as a result of AEs and one as a result of a drug-related cerebral hemorrhage. Within 30 days of discontinuation, 20 patients receiving enzastaurin died as a result of PD and three patients died as a result of AEs (one as a result of drug-related cerebral hemorrhage). In the lomustine arm, all four deaths $(4.8 \%)$ on study were disease-related. Eleven patients treated with lomustine died within 30 days of discontinuation (10 as a result of PD and one as a result of $\mathrm{AE}$ ).

\section{Postdiscontinuation Therapy}

Of the 70 patients treated with enzastaurin who received further salvage treatment, $\geq 5 \%$ of patients received lomustine $(12.1 \%)$, 


\begin{tabular}{|c|c|c|c|c|c|c|c|c|}
\hline \multirow[b]{3}{*}{ Toxicity } & \multicolumn{4}{|c|}{$\begin{array}{l}\text { Enzastaurin } \\
(\mathrm{n}=167) \dagger\end{array}$} & \multicolumn{4}{|c|}{$\begin{array}{l}\text { Lomustine } \\
(\mathrm{n}=84) \dagger\end{array}$} \\
\hline & \multicolumn{2}{|c|}{ Grade 2} & \multicolumn{2}{|c|}{$\begin{array}{c}\text { Grade } 3 \\
\text { to } 4 \\
\end{array}$} & \multicolumn{2}{|c|}{ Grade 2} & \multicolumn{2}{|c|}{$\begin{array}{c}\text { Grade } 3 \\
\text { to } 4\end{array}$} \\
\hline & No. & $\%$ & No. & $\%$ & No. & $\%$ & No. & $\%$ \\
\hline \multicolumn{9}{|l|}{ Nonhematologic toxicity } \\
\hline Fatigue & 5 & 3.0 & 6 & 3.6 & 5 & 6.0 & 0 & \\
\hline Anorexia & 2 & 1.2 & 0 & & 0 & & 0 & \\
\hline Nausea & 1 & 0.6 & 0 & & 2 & 2.4 & 0 & \\
\hline GI & 2 & 1.2 & 0 & & 0 & & 0 & \\
\hline Flatulence & 2 & 1.2 & 0 & & 0 & & 0 & \\
\hline Edema & 7 & 4.2 & 2 & 1.2 & 2 & 2.4 & 0 & \\
\hline Infection & 1 & 0.6 & 2 & 1.2 & 1 & 1.2 & 3 & 3.6 \\
\hline Stomatitis & 2 & 1.2 & 0 & & 2 & 2.4 & 0 & \\
\hline Thrombosis & 1 & 0.6 & $3 \neq$ & 1.8 & 0 & & 0 & \\
\hline \multicolumn{9}{|l|}{ Hematologic toxicity } \\
\hline Anemia & 1 & 0.6 & 0 & & 3 & 3.6 & 2 & 2.4 \\
\hline Neutropenia & 1 & 0.6 & 0 & & 4 & 4.8 & 17 & 20.2 \\
\hline Thrombocytopenia & 4 & 2.4 & 1 & 0.6 & 9 & 10.7 & 21 & 25.0 \\
\hline Lymphopenia & 0 & & 0 & & 2 & 2.4 & 0 & \\
\hline Leukopenia & 2 & 1.2 & 0 & & 3 & 3.6 & 6 & 7.1 \\
\hline \multicolumn{9}{|c|}{$\begin{array}{l}\text { "Grade } 2 \text { or grade } 3 \text { to } 4 \text { toxicities in one or more patient in either arm. } \\
+15 \text { patients (seven assigned to enzastaurin, eight assigned to lomustine) } \\
\text { discontinued before receiving treatment and were not included in the } \\
\text { safety analysis. } \\
\text { ¥Two patients receiving enzastaurin had grade } 4 \text { thrombosis. }\end{array}$} \\
\hline
\end{tabular}

bevacizumab (9.8\%), irinotecan (9.1\%), and carmustine $(5.7 \%)$. Of the 40 patients treated with lomustine who received salvage treatment, $\geq 5 \%$ of patients received carboplatin $(9.8 \%)$, temozolomide $(9.8 \%)$, irinotecan $(6.5 \%)$, lomustine (5.4\%), etoposide (5.4\%), and bevacizumab (5.4\%).

\section{Pharmacokinetics}

Enzastaurin plasma concentration data were available from 115 patients on day 21 of cycle 1 and from 11 patients on day 1 of cycle 4 . Steady-state plasma concentrations of enzastaurin and its metabolite LY326020 were within the range seen previously in patients with solid tumors. ${ }^{26}$

\section{DISCUSSION}

This is the first phase III trial evaluating a targeted therapeutic agent for the treatment of recurrent glioblastoma. The interim analysis served to determine whether enzastaurin was superior to lomustine and whether it was appropriate to continue enrollment. Because enzastaurin did not demonstrate superior PFS, enrollment was stopped. The final analysis showed no significant difference in any of the efficacy end points.

PFS was selected as the primary end point because previous studies indicated that the 6-month PFS rate was an appropriate end point for recurrent disease. ${ }^{34}$ PFS observed in the present study was similar to data obtained from other studies of targeted agents in recurrent glioblastoma, ${ }^{35-37}$ with the exception of other antiangiogenic targeted therapies, in particular, bevacizumab. ${ }^{20,38,39}$ However, in contrast to cediranib and bevacizumab, the anti-VEGF effects at- tributed to enzastaurin ${ }^{15}$ were not seen in this trial. Also, the overall response was lower.

Given the challenge of measuring progression in recurrent glioblastoma, we used modified Levin's response criteria, ${ }^{29}$ as in the phase II study. ${ }^{28,40}$ Response in gliomas is commonly assessed using Macdonald's criteria, ${ }^{41}$ a derivative of Levin's criteria; however, there has never been a formal comparison between the two. Most recently, Levin's criteria has been shown to be more predictive for response to antiangiogenic treatments than Macdonald's criteria. ${ }^{42}$ Many patients with recurrent glioblastoma have lesions without clearly defined margins or have a cystic component and are therefore not amenable to bidirectional measurement. In this study, progression of such lesions was defined as an increase in the enhancing component on MRI, as determined by the institutional radiologist. An independent review of the scans from a subset of patients, using both study criteria and Macdonald criteria, did not show a difference in PFS. Furthermore, deterioration in patient-reported outcomes was consistent with PFS.

Baseline characteristics were balanced between treatment arms. There was no significant treatment interaction between age or time from diagnosis to random assignment and either PFS or OS.

In this study, enzastaurin was well tolerated. The most common AEs observed in the enzastaurin arm, such as fatigue and headache, are expected in patients with glioblastoma. It is important to note that patients in this trial were switched from EIAEDs to non-EIAEDs 14 days before the trial, which may explain the relatively high number of convulsions observed. Although more patients in the enzastaurin arm discontinued because of AEs, there were no significant differences in the incidence of serious AEs, except for neutropenia, which occurred in significantly fewer patients in the enzastaurin arm. Thrombocytopenia in one patient was the only grade 3 to 4 toxicity observed with enzastaurin. This incidence of thrombocytopenia was lower than that observed in the earlier phase II trial. ${ }^{40}$

In retrospect, the enthusiasm over data from the phase II trial ${ }^{28}$ led to the immediate initiation of this phase III trial. Although the final analysis of the phase II trial continued to show a relatively high objective response rate (ORR) of $26 \%$ for recurrent glioblastoma, the overall outcome of patients was poor, with a median PFS of 1.3 months, a 6- month PFS rate of 7\%, and a median OS of 4.4 months. ${ }^{40}$ The properly controlled phase III trial reported here did not produce an ORR similar to the phase II trial; however, both had similarly disappointing long-term outcomes.

To date, clinical trials with single-agent targeted therapies have been largely disappointing. Bevacizumab has demonstrated a high ORR (according to Macdonald's or Levin's criteria), clinical benefit, and a meaningful $\mathrm{PFS}^{38,42}$; however, the drug has not yet been tested in a controlled setting against another compound. On the other hand, whereas PFS was longer with single-agent bevacizumab compared with our trial, median OS was comparable ( 31 weeks). ${ }^{42}$ Given the heterogeneity of glioblastomas, well-controlled phase III trials will be required to conclusively demonstrate that any given treatment agent will provide a substantial survival benefit in recurrent glioblastoma. Combinatorial targeted therapy is likely to prove more successful than single-agent therapy. ${ }^{43}$ The low toxicity observed with enzastaurin and the modest efficacy in the present trial, combined with robust preclinical data, ${ }^{23,44}$ suggest that enzastaurin may be suitable for combination therapy. Clinical trials of combinations of enzastaurin with radiotherapy, temozolomide, carboplatin, and bevacizumab are ongoing. ${ }^{44,45}$ 


\section{AUTHORS' DISCLOSURES OF POTENTIAL CONFLICTS} OF INTEREST

Although all authors completed the disclosure declaration, the following author(s) indicated a financial or other interest that is relevant to the subject matter under consideration in this article. Certain relationships marked with a "U" are those for which no compensation was received; those relationships marked with a " $C$ " were compensated. For a detailed description of the disclosure categories, or for more information about ASCO's conflict of interest policy, please refer to the Author Disclosure Declaration and the Disclosures of Potential Conflicts of Interest section in Information for Contributors.

Employment or Leadership Position: Shengyan Hong, Eli Lilly (C); Luna Musib, Eli Lilly (C); Astra M. Liepa, Eli Lilly (C); Donald E. Thornton, Eli Lilly (C) Consultant or Advisory Role: Wolfgang Wick, Eli Lilly (C); Vinay K. Puduvalli, Merck (C); Marc C. Chamberlain, Enzon (U), Genentech (U); Martin J. van den Bent, Eli Lilly (C); Lawrence M. Cher, Schering-Plough (C); Warren Mason, Eli Lilly (C); Michael Weller, Eli Lilly (C) Stock Ownership: Shengyan Hong, Eli Lilly; Luna Musib, Eli Lilly; Astra M. Liepa, Eli Lilly; Donald E. Thornton, Eli Lilly Honoraria: Vinay K. Puduvalli, Merck; Marc C. Chamberlain, Enzon, Genentech Research Funding: Vinay K. Puduvalli, Merck, Celgene, Pfizer, Eli Lilly; Lawrence M. Cher, Schering-Plough, Eli Lilly,
Merck, AstraZeneca Expert Testimony: None Other Remuneration: Lawrence M. Cher, Schering-Plough

\section{AUTHOR CONTRIBUTIONS}

Conception and design: Michael Weller, Astra M. Liepa, Donald E. Thornton, Howard A. Fine

Administrative support: Michael Weller

Provision of study materials or patients: Wolfgang Wick, Vinay K. Puduvalli, Marc C. Chamberlain, Martin J. van den Bent, Antoine F. Carpentier, Lawrence M. Cher, Warren Mason, Michael Weller Collection and assembly of data: Wolfgang Wick, Marc C. Chamberlain, Warren Mason, Howard A. Fine

Data analysis and interpretation: Wolfgang Wick, Marc C. Chamberlain, Shengyan Hong, Luna Musib, Astra M. Liepa, Donald E. Thornton, Howard A. Fine

Manuscript writing: Wolfgang Wick, Vinay K. Puduvalli, Marc C. Chamberlain, Martin J. van den Bent, Antoine F. Carpentier, Lawrence M. Cher, Michael Weller, Shengyan Hong, Luna Musib, Astra M. Liepa, Donald E. Thornton, Howard A. Fine

Final approval of manuscript: Wolfgang Wick, Vinay K. Puduvalli, Marc C. Chamberlain, Martin J. van den Bent, Antoine F. Carpentier, Lawrence M. Cher, Warren Mason, Michael Weller, Shengyan Hong, Luna Musib, Astra M. Liepa, Donald E. Thornton, Howard A. Fine

\section{REFERENCES}

1. Behin A, Hoang-Xuan $K$, Carpentier AF, et al: Primary brain tumours in adults. Lancet 361:323331, 2003

2. DeAngelis LM: Chemotherapy for brain tumors: A new beginning. N Engl J Med 352:10361038, 2005

3. Grossman SA, Batara JF: Current management of glioblastoma multiforme. Semin Oncol 31 635-644, 2004

4. Shapiro WR, Green SB, Burger PC, et al: Randomized trial of three chemotherapy regimens and two radiotherapy regimens in postoperative treatment of malignant glioma. Brain Tumor Cooperative Group Trial 8001. J Neurosurg 71:1-9, 1989

5. Fine HA, Dear KB, Loeffler JS, et al: Metaanalysis of radiation therapy with and without adjuvant chemotherapy for malignant gliomas in adults. Cancer 71:2585-2597, 1993

6. Stupp R, Mason WP, van den Bent MJ, et al: European Organisation for Research and Treatment of Cancer Brain Tumor and Radiotherapy Groups; National Cancer Institute of Canada Clinical Trials Group: Radiotherapy plus concomitant and adjuvant temozolomide for glioblastoma. N Engl J Med 352 987-996, 2005

7. Levin VA, Wilson CB: Nitrosourea chemotherapy for primary malignant gliomas. Cancer Treat Rep 60:719-724, 1976

8. Wong ET, Hess KR, Gleason MJ, et al: Outcomes and prognostic factors in recurrent glioma patients enrolled onto phase II clinical trials. J Clin Oncol 17:2572-2578, 1999

9. Chang SM, Theodosopoulos P, Lamborn K, et al: Temozolomide in the treatment of recurrent malignant glioma. Cancer 100:605-611, 2004

10. Wick A, Felsberg J, Steinbach JP, et al: Efficacy and tolerability of temozolomide in an alternating weekly regimen in patients with recurrent glioma. J Clin Oncol 25:3357-3361, 2007

11. Rosenthal MA, Ashley DL, Cher L: BCNU as second line therapy for recurrent high-grade glioma previously treated with temozolomide. J Clin Neurosci 11:374-375, 2004

12. Plate $\mathrm{KH}$, Breier $\mathrm{G}$, Weich $\mathrm{HA}$, et al: Vascular endothelial growth factor and glioma angiogenesis: Coordinate induction of VEGF receptors, distribution of VEGF protein and possible in vivo regulatory mechanisms. Int J Cancer 59:520-529, 1994

13. Berkman RA, Merrill MJ, Reinhold WC, et al: Expression of the vascular permeability factor/vascular endothelial growth factor gene in central nervous system neoplasms. J Clin Invest 91:153-159, 1993

14. Lamszus $\mathrm{K}$, Ulbricht $U$, Matschke $J$, et al: Levels of soluble vascular endothelial growth factor (VEGF) receptor 1 in astrocytic tumors and its relation to malignancy, vascularity, and VEGF-A. Clin Cancer Res 9:1399-1405, 2003

15. Yoshiji H, Kuriyama S, Ways DK, et al: Protein kinase $\mathrm{C}$ lies on the signaling pathway for vascular endothelial growth factor-mediated tumor development and angiogenesis. Cancer Res 59:4413-4418, 1999

16. Livneh E, Fishman DD: Linking protein kinase C to cell-cycle control. Eur J Biochem 248:1-9, 1997

17. Sano $T$, Lin $H$, Chen $X$, et al: Differential expression of MMAC/PTEN in glioblastoma multiforme: Relationship to localization and prognosis. Cancer Res 59:1820-1824, 1999

18. Stambolic V, Suzuki A, de la Pompa JL, et al: Negative regulation of PKB/Akt-dependent cell survival by the tumor suppressor PTEN. Cell 95:29-39, 1998

19. Koul D, Parthasarathy $R$, Shen $R$, et al: Suppression of matrix metalloproteinase-2 gene expression and invasion in human glioma cells by MMAC/ PTEN. Oncogene 20:6669-6678, 2001

20. Vredenburgh JJ, Desjardins A, Herndon JE II, et al: Phase II trial of bevacizumab and irinotecan in recurrent malignant glioma. Clin Cancer Res 13: 1253-1259, 2007

21. Reardon $D$, Fink K, Mikkelsen $T$, et al: Randomized phase II study of cilengitide, an integrintargeting arginine-glycine-aspartic acid peptide, in recurrent glioblastoma multiforme. J Clin Oncol 26: 5610-5617, 2008
22. Raizer JJ, Abrey LE, Wen P, et al: A phase II trial of erlotinib (OSI-774) in patients (pts) with recurrent malignant gliomas (MG) not on EIAEDs. J Clin Oncol 22:107s, 2004 (suppl; abstr 1502)

23. Graff JR, McNulty AM, Hanna KR, et al: The protein kinase Cbeta-selective inhibitor, enzastaurin (LY317615.HCl), suppresses signaling through the AKT pathway, induces apoptosis, and suppresses growth of human colon cancer and glioblastoma xenografts. Cancer Res 65:7462-7469, 2005

24. Keyes KA, Mann L, Sherman $M$, et al: LY317615 decreases plasma VEGF levels in human tumor xenograft-bearing mice. Cancer Chemother Pharmacol 53:133-140, 2004

25. Teicher BA, Alvarez E, Menon K, et al: Antiangiogenic effects of a protein kinase Cbeta-selective small molecule. Cancer Chemother Pharmacol 49: 69-77, 2002

26. Carducci MA, Musib L, Kies MS, et al: Phase I dose escalation and pharmacokinetic study of enzastaurin, an oral protein kinase $\mathrm{C}$ beta inhibitor, in patients with advanced cancer. J Clin Oncol 24: 4092-4099, 2006

27. Watkins V, Hong S, Lin B: Enzastaurin safety review: Data from phase I and phase II trials. J Clin Oncol 24:608s, 2006 (suppl; abstr 13077)

28. Fine HA, Kim L, Royce $C$, et al: Results from phase II trial of Enzastaurin (LY317615) in patients with recurrent high grade gliomas. J Clin Oncol 23:115s, 2005 (suppl; abstr 1504)

29. Levin VA, Crafts DC, Norman DM, et al: Criteria for evaluating patients undergoing chemotherapy for malignant brain tumors. J Neurosurg 47:329-335, 1977

30. Weitzner MA, Meyers CA, Gelke CK, et al: The Functional Assessment of Cancer Therapy (FACT) scale: Development of a brain subscale and revalidation of the general version (FACT-G) in patients with primary brain tumors. Cancer 75:11511161, 1995

31. Beaumont $\mathrm{JL}$, Victorson $\mathrm{D}$, Peterman $\mathrm{AH}$, et al: Validation of Functional Assessment of Cancer Therapy-Brain (FACT-Br) in patients with recurrent high-grade gliomas. Qual Life Res A-78, 2006 (abstr 1356) 
32. van den Bent MJ, Wick W, Liepa AM, et al: Time to deterioration in dimensions of health-related quality of life (HRQL) for patients with recurrent glioblastoma multiforme: Results from the randomized, phase III trial of enzastaurin (ENZ) versus lomustine (CCNU). J Clin Oncol 26:99s, 2008 (suppl; abstr 2040)

33. Oakes D: A concordance test for independence in the presence of censoring. Biometrics 38:451-455, 1982

34. Ballman KV, Buckner JC, Brown PD, et al: The relationship between six-month progression-free survival and 12-month overall survival end points for phase II trials in patients with glioblastoma multiforme. Neuro Oncol 9:29-38, 2007

35. Rich JN, Reardon DA, Peery T, et al: Phase II trial of gefitinib in recurrent glioblastoma. J Clin Oncol 22:133-142, 2004

36. Raymond E, Brandes AA, Dittrich C, et al: Phase II study of imatinib in patients with recurrent gliomas of various histologies: A European Organi- sation for Research and Treatment of Cancer Brain Tumor Group Study. J Clin Oncol 26:4659-4665, 2008

37. Gerstner ER, Duda DG, di Tomaso E, et al: Antiangiogenic agents for the treatment of glioblastoma. Expert Opin Investig Drugs 16:1895-1908, 2007

38. Cloughesy TF, Prados MD, Wen PY, et al: A phase II, randomized, non-comparative clinical trial of the effect of bevacizumab (BV) alone or in combination with irinotecan (CPT) on 6-month progression free survival (PFS6) in recurrent, treatment-refractory glioblastoma (GBM). J Clin Oncol 26:91s, 2008 (suppl, abstr 2010b)

39. Batchelor TT, Sorensen AG, di Tomaso E, et al: AZD2171, a pan-VEGF receptor tyrosine kinase inhibitor, normalizes tumor vasculature and alleviates edema in glioblastoma patients. Cancer Cell 11:83-95, 2007

40. Kreisl TN, Kotliarova S, Butman J, et al: A phase $|/| I$ trial of enzastaurin in patients with recurrent high-grade gliomas. Neuro-Oncology (in press)
41. Macdonald DR, Cascino TL, Schold SC Jr, et al: Response criteria for phase II studies of supratentorial malignant glioma. J Clin Oncol 8:12771280, 1990

42. Kreisl TN, Kim L, Moore K, et al: Phase II trial of single-agent bevacizumab followed by bevacizumab plus irinotecan at tumor progression in recurrent glioblastoma. J Clin Oncol 27:740-745, 2009

43. Mischel PS, Cloughesy TF: Targeted molecular therapy of GBM. Brain Pathol 13:52-61, 2003

44. Tabatabai G, Frank B, Wick A, et al: Synergistic anti-glioma activity of radiotherapy and enzastaurin. Ann Neurol 61:153-161, 2007

45. Butowski NA, Lamborn K, Chang $S$, et al: Phase I/II study of enzastaurin (ENZ) plus temozolomide (TMZ) and radiation therapy (XRT) in patients with glioblastoma multiforme (GBM) or gliosarcoma (GS). J Clin Oncol 26:167s, 2008 (suppl; abstr 3559)

\section{Sign up for Alerts About Your Topic of Interest}

Learn about new research in your field as it becomes available. Subscribe to a JCO e-mail alert to be notified immediately when new articles within your area of interest are posted.

Receive notification when:

$>J C O$ releases a new issue's Table of Contents.

$>$ A new issue of $J C O$ is posted online.

$>$ New articles are published online ahead of print publication.

$>$ New content in your subspecialty is published.

$>$ An article is published online from an author of interest.

Go to jco.org/alerts to sign up. 